\title{
Development of an HPC Enhanced Multi Agent Simulation Code for Tsunami Evacuation
}

\author{
Rithika Dulam ${ }^{1)}$, Maddegedara Lalith ${ }^{2)}$, Muneo Hori ${ }^{3)}$, Tsuyoshi Ichimura ${ }^{4)}$ and Seizo Tanaka ${ }^{5)}$ \\ 1) Master Student, Earthquake Research Institute, University of Tokyo, Japan \\ 2) Asst. Professor, Global Center of Excellence for Sustainable Urban Regeneration, University of Tokyo, Japan \\ 3) Professor, Earthquake Research Institute, University of Tokyo, Japan \\ 4) Assoc. Professor, Earthquake Research Institute, University of Tokyo, Japan \\ 5) Asst. Professor, Earthquake Research Institute, University of Tokyo, Japan
}

\begin{abstract}
We developed a multi agent simulation code, enhanced with high performance computing (HPC), for simulating mass evacuations triggered by catastrophic events like a tsunami. Prediction of mass evacuations is of great importance to ensure public safety, in which the complex human behaviour is a key issue. A parallel multi agent simulation code is developed with the aim studying the problems associated with large urban area evacuation; no real experiment is feasible for large area evacuation involving millions of people. The HPC enhanced code scales linearly up to $256 \mathrm{CPU}$ cores. A preliminary study is conducted to identify the effectiveness of reducing the evacuation time with official agents with different responsibilities. It was found that using 3\% officials produces similar results when all the agents evacuate immediately, without any preparation time.
\end{abstract}

Key Words: multi agent simulation, tsunami, pre-evacuation time, parallel programming.

\section{Introduction}

Prediction of mass evacuation in large urban areas, due to natural disasters like earthquakes, tsunami, etc., is of great importance to ensure public safety. Such studies have wide applications like finding strategies for saving lives in time for critical events like tsunami, modifying built environment to facilitate fast evacuation, city planning, training rescue officials, etc. Real experiments are not feasible for large area evacuation involving millions of people and small area experiments do not render a correct picture due to contagious and cascade effects of mass evacuation. Out of a number of numerical models like cellular automata, social force model, etc., Multi Agent System (MAS) is suited for modeling heterogeneous and complex human behavior involved in mass evacuations. Simulating millions of people in a large urban area with smart and sophisticated agents is computationally intensive. The need of Monte-Carlo simulations to improve the reliability of solutions drastically increases the amount of computation. Therefore, large urban area evacuation simulation requires high performance computing.

Pedestrian evacuation simulations can be classified into macroscopic and microscopic level simulations. Macroscopic models are based on analogy of pedestrians and fluids ${ }^{1)}$. There are number of methods used for microscopic level simulation of pedestrian dynamics; social force model, cellular automata, autonomous multi agents, etc. As the name implies, in the social force model, the internal motive of a pedestrian is modeled with social forces or social fields ${ }^{2}$. A main problem in the social force model is that people don't follow the laws of physics. Cellular automata method divides the domain into discrete cells, which typically holds a single pedestrian. Certain rules are used to compute the state of each dell as a function of the previous state and the state of adjacent cells ${ }^{3)}$. A weakness in cellular automata is that it cannot model dense crowd realistically. In autonomous multi agent approaches, each pedestrian is modeled as independent agent who can take spontaneous actions interacting with his surrounding and the environment ${ }^{4)}$. Properly designing to mimic human behaviors, autonomous multi agent approach can reproduce complicated patterns of real heterogeneous human crowds, which includes people of different abilities, knowledge, levels of cognition, responsibilities, etc. Because of its clear advantages, multi agent systems are considered in this study. 
Simulation of large urban area with smart and sophisticated agents requires high performance computing. Most of the HPC enhanced large scale pedestrian evacuation simulations based on MAS are limited to several ten thousands of agents and a few tens of $\mathrm{CPUs}^{5), 6)}$. In their proposal of distributed load balancing scheme, Cozenza et al. has demonstrated simulation of 100,000 agents $^{6}$. However, its scalability us limited a few tens of CPUs.

This work on HPC enhanced large urban area evacuation simulation is a part of much larger effort of developing an Integrated Earthquake Simulator (IES). IES is a system to seamlessly simulate earthquake hazard, induced disasters and the aftermaths, in large urban areas like Tokyo ${ }^{7}$. The existing MAS module is developed for simulating emergency evacuation of crowded small areas like shopping malls, train stations etc. Its agents have limited abilities, which are insufficient for autonomous navigation in complex urban areas and model complex human behaviors involved in emergency evacuations.

We improved the MAS module of IES by improving some of agent's abilities and implementing a high performance computing extension, with the aim of simulating large urban area evacuation. Agent's visual abilities, to see the environment, and decision making, to identify available paths and autonomously navigate in complex urban environment, are improved. In addition, different types of agents, to play the role of residents, officials, etc., with mutual interacting abilities are introduced. Further, it is enhanced with a scalable parallel computing extension, which significantly increased the problem size from several thousand agents in smaller domain to several millions of agents in a large domain. To demonstrate the capabilities of the improved MAS module, we conducted a preliminary study to find effectiveness of employing officials like police officers to reduce the evacuation time, in tsunami prone coastal areas.

The rest of the paper is organized as follows. The second section presents details of the design of agents and the environment. Strategies implemented in the scalable parallel computing extension are presented in the third section. The problem settings and the results of preliminary study are given in the fourth and the fifth sections.

\section{Methodology: multi agent systems}

MAS can be used to solve complex problems in diverse fields in computer science, marketing, social science etc. Multi agent systems consist of two main parts; agents and environment.

\subsection{Agent}

Agents take spontaneous actions when it interacts with other agents and its surrounding environment. Properly designing the agents and environment to mimic the elements of the problem in consideration is required. People have different amount of information and different level of mental and physical abilities. This heterogeneity generates complex patterns. Studying such complex patterns appear in mass evacuations is useful to identify and evaluate the effectiveness of various strategies for saving human lives. To that end, agents are designed with different visual, decision making and moving abilities.

\subsection{Environment}

The environment of our MAS is the model describing the configuration of a public space, in which the agents move. The environment is modeled as a structured grid, of cell size $1 \mathrm{~m}^{2}$, consisting of obstacle cells and open cells. It is automatically generated from GIS and CAD data (see Fig. 1). The agents can move around in the open cells avoiding the obstacles and collision.

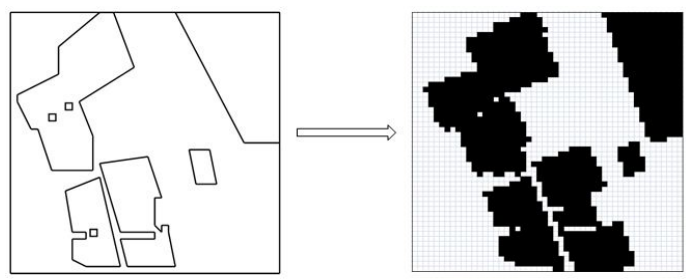

Fig. 1 The environment is generated with automated conversion of GIS data to grid data.

\subsection{Agent behavior in open environments}

The agent in the model has three basic functions, namely See(), Think() and Move(), which represent the human's most important features. The functions, which are self explanatory, are the vital aspects of the simulation. The agents of the former MAS code are designed based on the popular KISS, Keep It Simple and Stupid principle. KISS based agents are sufficient for simulating evacuation in crowded and simple environments. However, simulation of evacuation in large and complex urban environments requires smart and sophisticated agents with better vision and decision making abilities.

The vision and the decision making abilities are improved so that agents can navigate smoothly in complex urban environments. In the former code, the area influencing the decision, e.g. visual 
distance, is restricted to a very small radius of $1.5 \mathrm{~m}$, since larger radius is insignificant in crowded environments. The vision radius is increased to $30 \mathrm{~m}$ so that the agents can identify various features of their surroundings and navigate smoothly in urban areas. A good and compatible vision and decision making model assures a smooth agent motion, a key for human modeling.

\section{(1) Agent vision model}

Visual Perception is an important function of the agent model as the Think() function just analyzes the information generated by the vision. The vision radius is set to $30 \mathrm{~m}$, in the current settings. An agent scans the $360^{\circ}$ vision field at $d \theta$ intervals, similar to radar, and collects the grid cells in the line of sight. The value of $d \theta$ is set such that

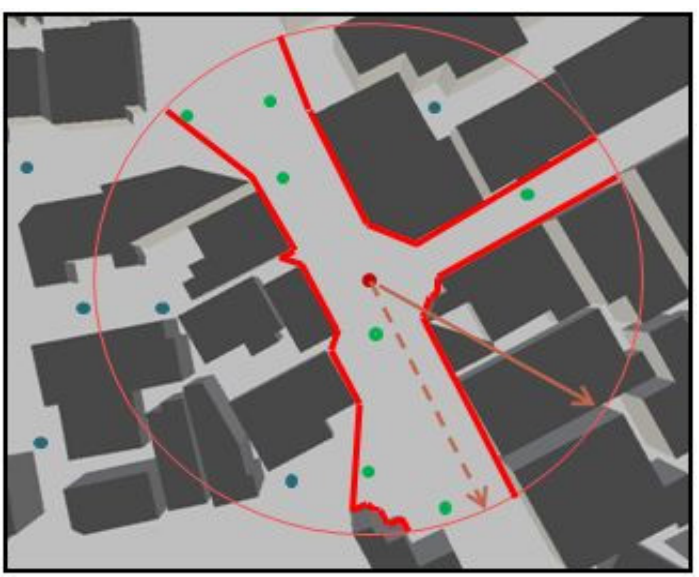

Fig. 2 Vision perception of an agent: red dots are the visible agent; thick red lines indicate the edge of visibility; direction of destination and movements are shown by the continuous and the dotted arrows

arc length with visibility radius is $50 \%$ of the grid size (i.e. visibility radius $\times d \theta=50 \%$ of the size of an grid element). For $30 \mathrm{~m}$ visibility radius, $d \theta$ is set to $1^{\circ}$. Currently, the agent vision is only horizontal, hence the elevation of the cell is not considered. Fig. 2 shows the vision of the agent and how it perceives the environment.

\section{(2) Decision making model}

The pivot of the agent model is the decision making function which is autonomous. In function Think(), it analyzes the data collected through the vision model and takes a decision, depending on its surroundings and the final destination. The agent scans through the view data, collects all the available paths and chooses the closest path to the final destination vector. Currently, the decision making is quite simple as it is still in its development stage.

\subsection{Movement in Grid environment}

The environment can be modeled either as vector or grid data. Vector type environment, which model the road network environment as a network of nodes connected with links (see Fig. 3), is popular among MAS based evacuation or pedestrian dynamic simulations ${ }^{8}$. Tracking an agent's path and not allowing an agent to re-trace a former taken path can be easily managed in such an environment as the path is a set of nodes and links. Autonomous navigation in grid environment is difficult, as identifying the features like the shape of buildings, junctions or roads is complicated. The agent can only recognize the open cells and obstacle cells; it is difficult to prevent the agents moving towards dead ends or to block them when moving towards already explored areas. There are many possibilities that an agent loops around in an area for a long time due to the complexity of the urban environment. Such agents are identified and $A^{*}$ path finding algorithm is used to find the path to move out of the trapped area. Once, an agent moves out of the trapped region, the fore mentioned Think() function resumes.

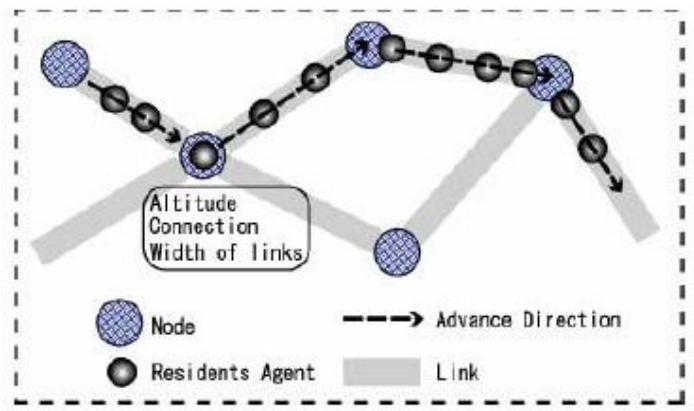

Fig. 3 Vector environment is a group of nodes and links

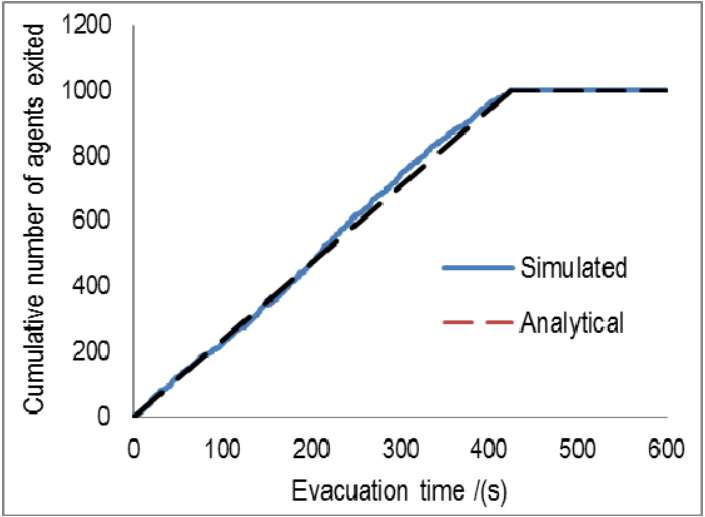

Fig. 4 Verification of the model 


\subsection{Verification of the model}

In the process of verifying the simulation results, we have considered the evacuation of 1000 agents in a grid tile, of $800 \times 600$ cells, without the obstacles or building data. It is essentially a 1 dimensional problem, as the agents move straight to the exit without travelling any extra distance. All the agents move at constant speed of $1.4 \mathrm{~m} / \mathrm{s}$. As shown in the Fig. 4, the simulated and analytical solutions are in good agreement. There is a slight deviation of the simulated results since distance to the exit is not ideally uniform at the beginning.

\section{Parallel Performance}

As suggested previously, for rendering a correct picture of the effects of mass evacuation, simulation of large numbers of people in a huge urban area is required. Simulating 30 minutes period of evacuation with 5000 people, in $0.5 \mathrm{~km}^{2}$ urban environment, requires around 10 hours on a single CPU. Hence, high performance computing resources have to be utilized to simulate large urban area. A distributed computing model with Message Passing Interface (MPI) is used in the current program. To balance the work load, the domain is partitioned such that each processor core is allotted approximately equal number of agents and the corresponding grid data. When an agent moves across the partition boundary, he is permanently moved to the corresponding CPU. Once significant load imbalance among the CPUs is observed, due to this agent movement, the domain is repartitioned to reassign balanced loads to the CPUs.

\subsection{Improving the Performance}

Existing MAS based crowd simulations have achieved limited scalability on small number of CPUs. With several strategies, we attained near ideal scalability up to $256 \mathrm{CPU}$ cores, with 250,000 agents. When the number of agents increased to 500,000 , the scalability curve is pushed to the super-linear region. The super-linear behavior is due to the non-linear reduction of neighbor search time with decreasing size of agents assigned to CPU cores. The main strategies used to attain this high scalability are: 1) virtual CPU topologies; 2) reducing the frequency of communications for ghost layer updates; 3 ) hiding the communications and minimizing the data volume; 4) minimize data exchange in repartitioning.

\section{(1) Virtual CPU topologies}

We use a 2D-tree algorithm to decompose the domain into square regions with nearly equal number of agents. Although this partitioning strategy does not minimize the communication data volume, the simple geometry makes it easier to handle agent movements between partitions. A major disadvantage of 2D-tree based partitioning is that we cannot take the advantage of communication topology of underlying hardware; the communication patterns between partitions are too irregular. However, the distributed graph topology interface of MPI 2.2 standard provides a more user friendly interface, to better match the communication pattern of the partitions.

\section{(2) Reduction of the frequency of ghost layer updates}

This is the main strategy for going beyond linear scalability, which is made possible by maintaining wide ghost regions (overlapping between two partitions). The human agents needs the ability to see far to detect obstacles, other agents and slow moving agent groups. This requires maintaining a wide ghost region, which should be larger than the visibility distance of 30 to $100 \mathrm{~m}$. Surely, this requirement significantly increases the communication overhead. However, in human crowd simulations, this wide ghost boundary can be used to gain high scalability. To explain, the terminology introduced in Fig. 5; a partition assigned to a CPU is further subdivided as shown. To avoid slow moving crowds, an agent needs to see the other agents at far end of his visibility. Therefore, a small error in the position of agents at the far edge of receive region does not affect the agents inside a partition. This allows skipping several ghost layer updates and maintaining the continuity by executing the agents in the ghost layer in a certain order along with the inner agents and boundary agents. The number of

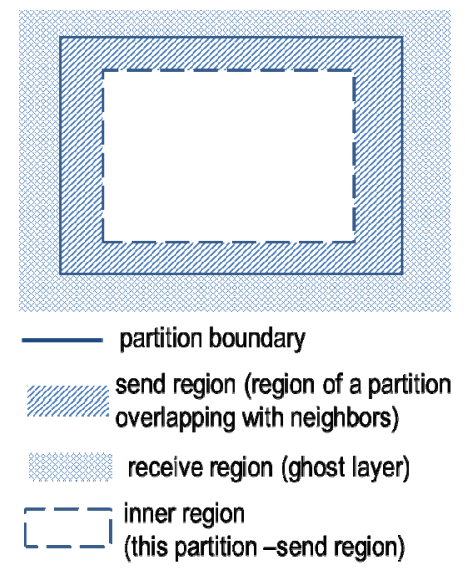

Fig. 5 Partitioning of the agents in each CPU 
steps to be skipped should be selected to minimize the propagation of any error into a partition from the agents at the far edge of receive region. Depending on the distance of agent's visibility, ghost updating at every 5-10 time steps is sufficient (time interval is $0.2 \mathrm{~s}$ ). This significantly increases the scalability of parallel extension of the evacuation module.

\section{(3) Hiding communications and minimizing} data volume exchanged

Compared with particle physics type simulations, of similar category, like SPH or N-body, sophisticated and smart agents involve 30 to 40 times the data. Further, sophisticated agents have dynamically growing variables like memory of past experiences. To deal with this large data volume, only the recent updates of dynamic data and essential data for ghost updating is exchanged during ghost update. Further, the ghost update communication is hidden by executing the agents in a certain order.

(4) Minimizing data exchange in repartitioning

Migration of agents from a partition to another brings load imbalance. When significant load imbalance occurs, repartition is necessary to maintain equal workloads. Repartitioning is a very expensive step since human agents have large sets of data. With 2D-tree, it is observed that most of the agents remain in the same CPU even after repartitioning unless the partitioning maps it to different CPUs. The repartitioning algorithm detects whether the same partition is assigned to a CPU and exchanges only the newly assigned agents, see Fig. 6. The point to note, here is that the interval should be such that it should not affect the movement of the boundary agents of the neighboring CPU. This drastically reduces the communication overhead in repartitioning, which lowers any performance degeneration due to repartitioning.

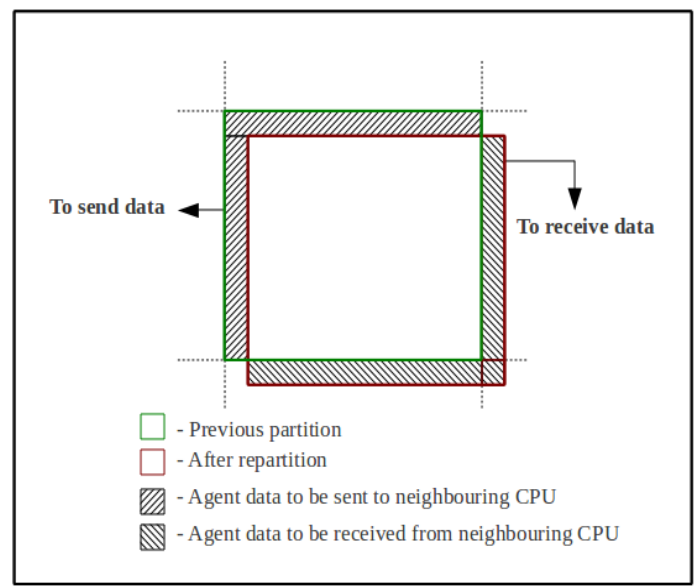

Fig. 6 Data exchange during repartitioning

\subsection{Scalability}

The effectiveness of the above major strategies was tested by conducting a series of simulations with 250,000 and 500,000 agents in a dense part of Kochi city, Japan, using up to $256 \mathrm{CPU}$ cores. A DELL cluster with QLogic 12200 InfiniBand switch and 16 computation nodes, each with hexa-core Intel Xeon X5680 CPUs and 47GB DDR3 memory, was used for the simulations. Fig. 7 and Fig. 8 show the simulation with 0.25 and 0.5 million agents and it can be observed that the obtained scalability is near linear and super-linear respectively. The super linear behavior is due to the nonlinear reduction of time for an agent to find its neighbors, when the number of agents in a CPU is halved. Fig. 8 shows high super-linear behavior since the larger the number of agents, the larger the reduction in neighbor search time.

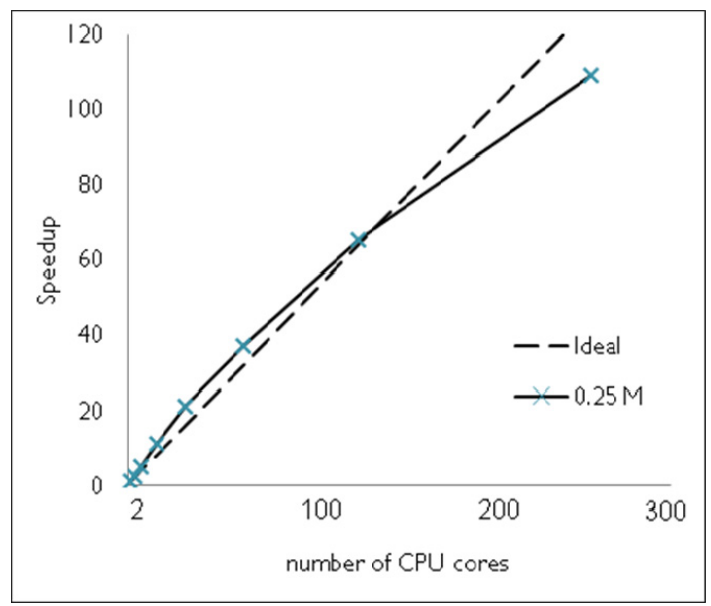

Fig. 7 Scalability with 250,000 agents

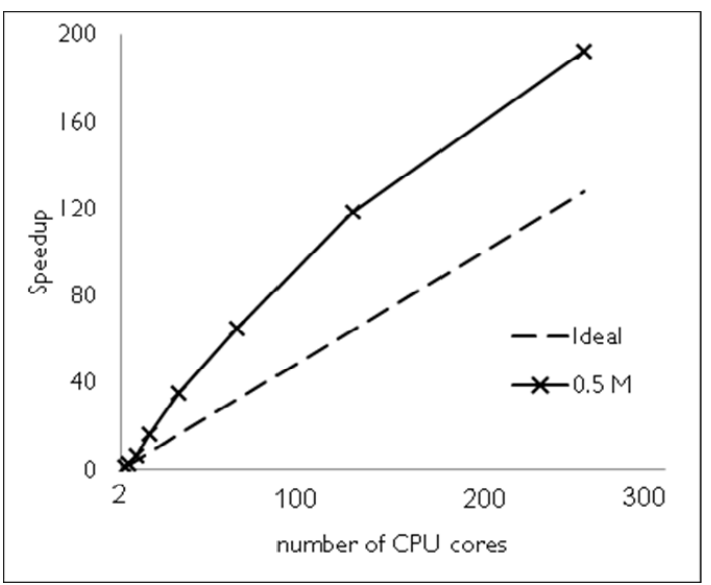

Fig. 8 Scalability with 500,000 agents 


\section{A preliminary study on tsunami evacuation time reduction: modeling of officials in a tsunami evacuation}

Reduction of the evacuation time is the key issue in tsunami disasters where the available time is less than 10 minutes for some areas. March 11, 2011 has seen a devastating picture of tsunami in Japan. According to a survey ${ }^{9)}$ conducted among the survivors of the 2011 Tohoku tsunami hit regions, between Yamamoto-cho and Minami-Sanriku-cho, pre-evacuation time is larger than 30 minutes, which is quite sufficient for a person to reach a safe zone. It has also been reported that comparatively less number of people heard warnings or messages from the local government or the local mitigation centers. The pre-evacuation time depends on many factors like perceived importance, uncertainty of the warning, finding family members, gathering valuables etc. Reducing this large pre-evacuation time might be one of the most effective means of reducing total evacuation time (pre-evacuation time + travel time), especially for areas with very short tsunami arrival times like for a tsunami induced by a possible Tokai-Tonankai-Nankai Earthquake. It is surely trivial to see that shorter preparation time is better. However, it is not straightforward to quantitatively analyze the effect of shortening the preparation time of the evacuation process.

Resorting to personalization would help reduce the evacuation time as it has been seen from the survey that many people ignore or do not hear the mass communication messages. Officials, like policemen or fire fighters, who have complete knowledge of their neighborhood, could be employed to personally instruct the people to evacuate and inform them about the nearest and safest evacuation center, depending on the intensity of the disaster. It is necessary to identify the effective number of officials for a given community, their distribution and legal strength in enforcing agents to evacuate.

\subsection{Officials and their functions}

The officials are smart agents with greater abilities, like the police, rescue officers, etc. They help people evacuate to safer areas. The main function of an the official is Guide() in which an official asks an agent to start evacuation immediately, using his influence power. Two types of officials were implemented, Type-A and Type-B. Type-A officials have less authority and less responsibility like volunteers; they help only the evacuees they find within a distance of influence radius, along their way towards the evacuation

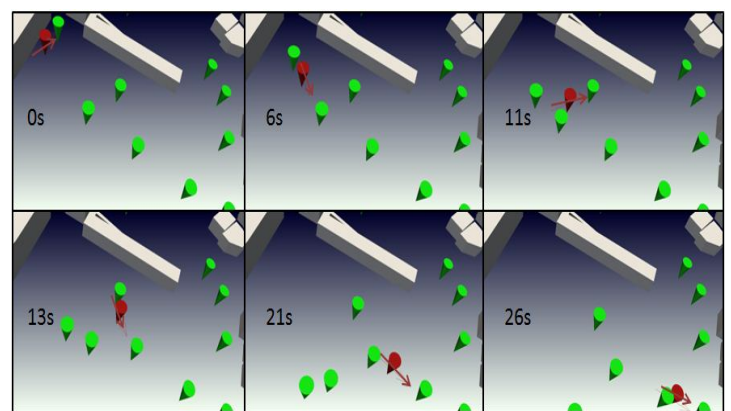

Fig. 9 Movement of a Type-B official

center. Type-B officials are more responsible and have more authority like the police officers; they search in their visibility for agents who have not started evacuation, move towards them and order to evacuate immediately. When the target agent is within the official's influence radius, the official informs him about the situation, the coming tsunami and suggests him to start evacuation immediately.

This influence radius is considered as the conversational distance. Various influence radius, $2.5 \mathrm{~m}$. $5.0 \mathrm{~m}, 10.0 \mathrm{~m}, 20.0 \mathrm{~m}, 30.0 \mathrm{~m}$ have been tested and it was found that there is no significant difference in $2.5 \mathrm{~m}$ and $10.0 \mathrm{~m}$; the extra $7.5 \mathrm{~m}$ travelled by the official to reach the agent is less significant. Therefore, a value of $2.5 \mathrm{~m}$ has been used for the simulation. In Fig. 9, the movement of a Type-B official is shown through the sequence of snapshots from left to right and top to down. We can see the official, the red cone, moving to each of the agents in his visibility and give them order to evacuate. The agents start to evacuate as soon as they receive the evacuation order from the official.

\section{Effectiveness in reducing pre-evacuation time of evacuees}

The effectiveness of the officials, in turn the ability of the model to produce the possible trends, is examined, explained as under.

\subsection{Problem Setting}

To examine the effectiveness of the officials in reducing the evacuation time, a number of simulations are conducted. Two domains of $0.48 \mathrm{~km}^{2}$ and $16.8 \mathrm{~km}^{2}$ from Kochi city, see Fig. 10, are used for these simulations. Agents move towards higher grounds, once they decide to evacuate. The basic parameters of the simulation are shown in Table 1. Average and standard deviation of agents' speeds are obtained from the reference 10 while average and standard deviation for prepara- 
tion time care calculated based on the survey conducted among the survivors of 2011 Tohoku tsunami ${ }^{9}$.

Table 1 Parameters used in the simulation

\begin{tabular}{|c|c|}
\hline Parameter & Values \\
\hline $\begin{array}{c}\text { Average moving speed of } \\
\text { Evacuees [m/s] } \\
\text { Normal } \\
\text { Slow }\end{array}$ & 1.4 \\
\cline { 2 - 2 } & 1.1 \\
\hline $\begin{array}{c}\text { Standard deviation of moving } \\
\text { speed of Evacuees [m/s] } \\
\text { Normal } \\
\text { Slow }\end{array}$ & 0.6 \\
\cline { 2 - 2 } Moving speed of officials[m/s] & 0.3 \\
\hline $\begin{array}{c}\text { Average preparation time [se- } \\
\text { conds] }\end{array}$ & 1000 \\
\hline $\begin{array}{c}\text { SD of preparation time [se- } \\
\text { conds] }\end{array}$ & 240 \\
\hline
\end{tabular}

The developed HPC enhanced MAS code can simulate millions of agents in large domain. However, a small problem of 1000 agents in an area of $0.48 \mathrm{~km}^{2}$ (a grid of size, $800 \times 600$ cells) is considered in this preliminary study. The density of officials was varied between $0 \%, 0.5 \%, 1 \%$, $3 \%, 5 \%$ and $10 \%$.

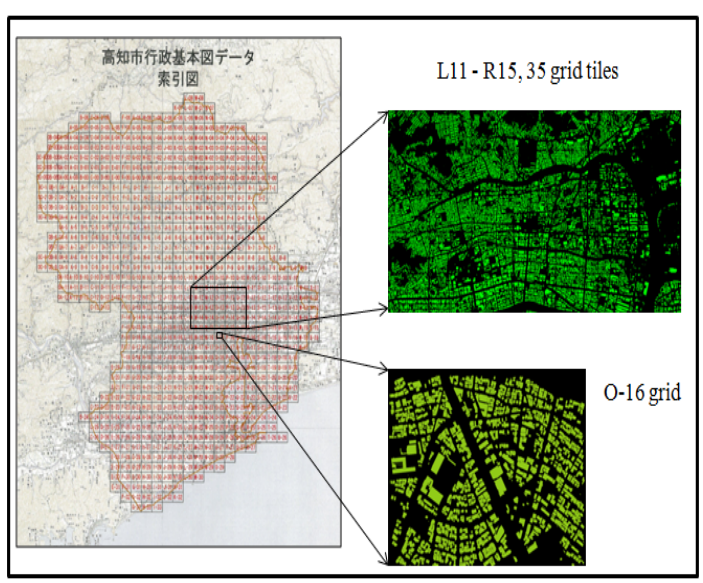

Fig. 10 Kochi City grids used as the environment for the simulation

\subsection{Results}

Fig. 11 presents the cumulative evacuation time with varying velocity and varying preparation time using Type-A officials. The curve without a caption is for the ideal case where preparation time is zero and the rest of the cases have varying preparation time with increasing number of officials from $0 \%, 1 \%, 3 \%, 5 \%$ and $10 \%$ respectively. Type-A officials were found to be not as effective as expected. The effect on the evacuation time was obtained with only more percent of Type-A officials.

Fig. 12 presents the cumulative evacuation time with Type-B officials. The curve without a caption is for the ideal case where preparation time is zero and the rest are with increasing number of officials from $0 \%, 0.5 \%, 1 \%, 3 \%, 5 \%$ and $10 \%$ respectively. We can observe that the usage of $0.5 \%$ officials has significant effect on the evacuation times of the agents. The irregularity of each curve shows the complexity of the problem, where more than one parameter, like speed, distribution of agents, preparation time and complexity of the environment, is in question. The evacuation time has shown considerable difference with every increase in number of officials. It can also be noted that the usage of $3 \%, 5 \%$ and $10 \%$ have obtained almost similar results, stating that there is a tendency of saturation.

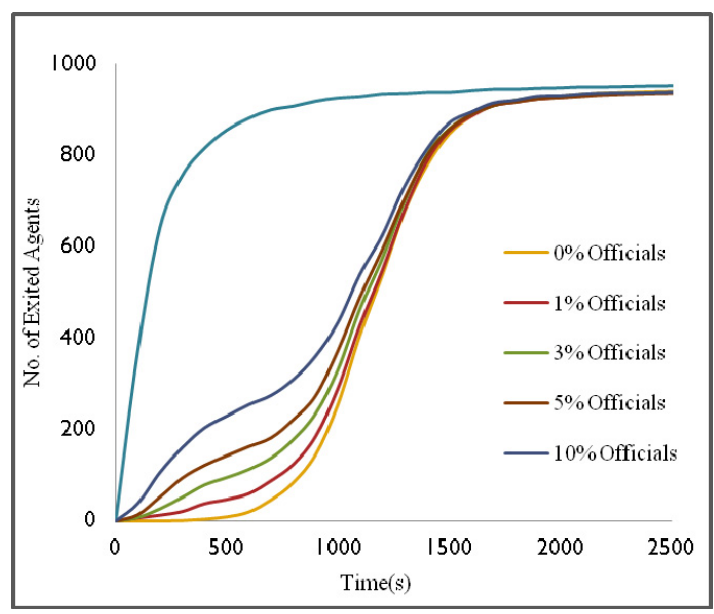

Fig. 11 Evacuation time with Type-A officials in O-16 domain (1000 agents)

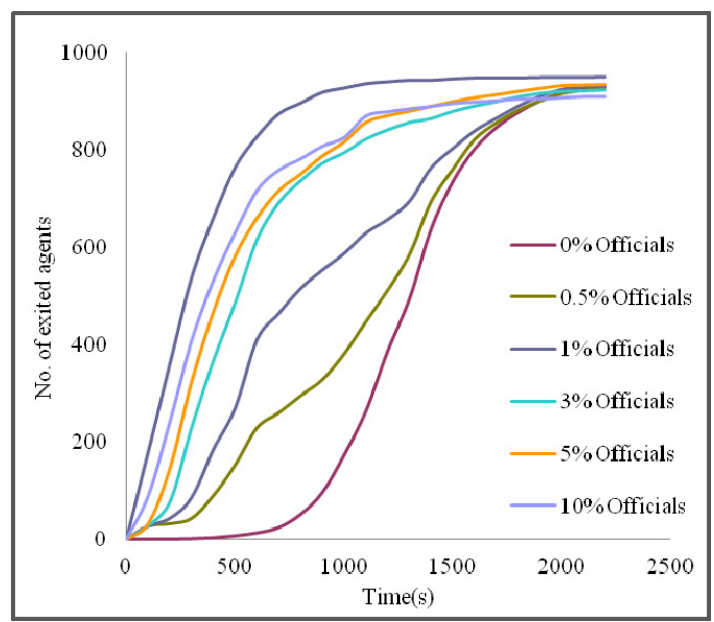

Fig. 12 Evacuation time with Type-B officials in O-16 domain (1000 agents) 


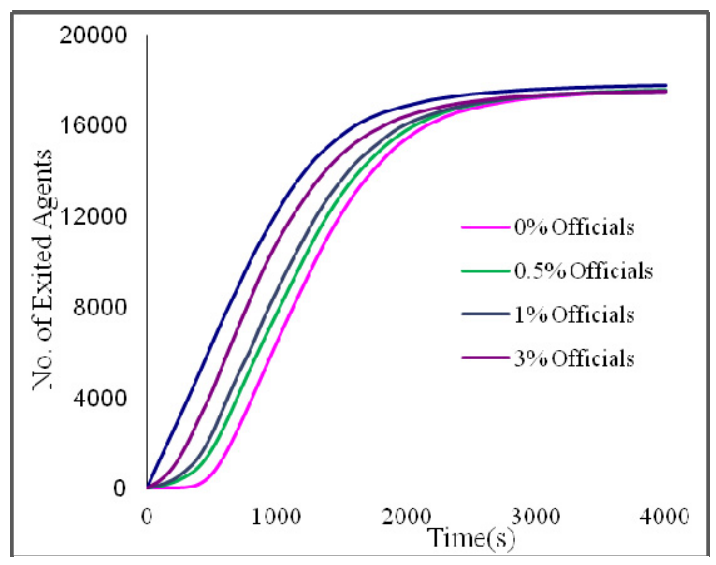

Fig. 13 Evacuation time with Type-B officials in L11 - R15 domain (20000 agents)

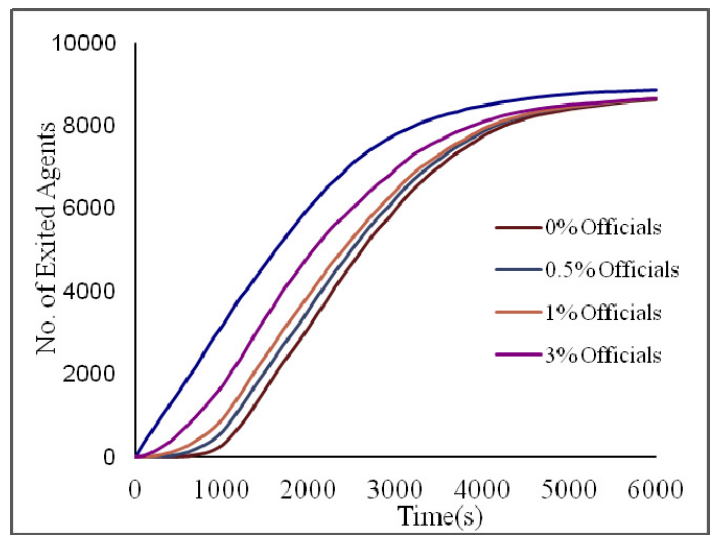

Fig. 14 Evacuation time with Type-B officials in L11- R15 domain (10000 agents)

We have considered a larger domain of 16.8 $\mathrm{km}^{2}$ with 35 grid tiles (L11 - R15) of Kochi city (see Fig. 10), with 10000 and 20000 agents respectively. The density of officials is increased from $0.5 \%, 1 \%, 3 \%$, as we have observed that $5 \%$ and $10 \%$ officials does not show much difference.

It can be observed from Fig. 13 and Fig. 14 that the behavior is similar to 1000 agents case (Fig. 12) with each increase of the percentage of officials. Hence, in a mass evacuation of 20000 people, it can be stated that employing officials has positive effects on reducing total evacuation time.

\section{Concluding Remarks}

An HPC enhanced MAS code is developed for simulating mass evacuation in a large urban area. A new KISS, Keep It Smart and Sophisticated principle was used to model the agents. To meet the computational demand of simulating large urban area with smart and sophisticated agents, the code is enhanced with distributed memory computing extension. With a number of strategies, near linear scalability is attained; with 0.25 million agents near linear and with 0.5 million agents super-linear scalability are attained on $256 \mathrm{CPU}$ cores.

A preliminary study is conducted to find the effectiveness of using officials to personally instruct the residents to evacuate immediately. The officials are employed to search for the agents who have not started evacuation and ask them to evacuate immediately. According to the Figs. 11-14, this official's behavior has significant effect of reduction of total evacuation time. An optimum level of $3 \%$ officials is found to show satisfactory results almost similar to the one with no preparation time.

\subsection{Future Work}

More effective reduction of evacuation time can be expected using officials with other factors like information passing, group behaviors or using Type-A and Type-B together which help in reducing the evacuation time. For more reliable results, the agent must be modeled to be more sophisticated to accommodate the necessary human behaviors, like group behavior, neighbor bonding etc. Human cognition has to be considered in modeling the pre-evacuation time, instead of the currently assigned random time.

\section{References}

1) Takahashi, K., Tanaka T. and S. Kose, An Evacuation Model For Use In Fire Safety Design Of Buildings. Proceedings of the Second International Symposium on FireSafety Science, pp. 551-560, 1989.

2) Helbing, D. and Molnár, P., Social force model for pedestrian dynamics. Physical Rev E, Vol. 51-5, pp. 4282-4286, 1995.

3) Blue, V. and Adler, J., Emergent fundamental pedestrian flows from cellular automata microsimulation. Transp. Res. Rec. 1644, pp. 29-36, 1998.

4) Farinelli, A., Grisetti, G., Iocchi, L., Lo Cascio, S. and Nardi, D., Using the RoboCup-Rescue Simulator in an Italian Earthquake Scenario, Proc. of the 1st International Workshop on Synthetic Simulation and Robotics to Mitigate Earthquake Disaster, Padua, Italy, 2003.

5) Vigueras, G., Lozano, M., Perez, C. and Orduna, J.M., A Scalable Architecture for Crowd Simulation: Implementing a Parallel Action Server, Proceedings of the 37th 
international Conference on Parallel Processing, IEEE Computer Society. pp. 430-437, 2008.

6) Biagio Cosenza, Gennaro Cordasco, Rosario De Chiara and Vittorio Scarano, Distributed Load Balancing for Parallel Agent-based Simulations, Proceedings of the 19th International Euromicro Conference on Parallel, Distributed and Network-based Processing, pp. 62-69, 2011.

7) Hori, M., Ichimura, T. and Oguni, K., Development of Integrated Earthquake Simulation for estimation of strong ground motion, structural responses and human actions in urban areas, Asian Journal of Civil
Engineering (building and Housing), Vol.7, No. 4, pp. 381-392, 2006.

8) Takashi Saito and Hiroshi Kagami: Simulation of evacuation behavior from tsunami utilizing multi agent system. $13^{\text {th }}$ World Conference on Earthquake Engineering, Vancouver, B.C, Canada. Paper No. 612, 2004.

9) 宮城県沿岸部における被災地アンケート 調査報告書 2011, Survey research center co., 2011.

10)Hori, M., Multi Agent Simulation for evacuation process analysis, Introduction to Computational Earthquake engineering, $2^{\text {nd }}$ edition, Chapter 14. Pp. 317-358, 2011.

(Received March 8, 2012) 